\title{
Conomics
}

The Open-Access, Open-Assessment E-Journal

Discussion Paper

No. 2011-28 | July 28, 2011 | http://www.economics-ejournal.org/economics/discussionpapers/2011-28

\section{A Directional-Change Events Approach for Studying Financial Time Series}

\author{
Monira Aloud \\ School of Computing and Electronic Systems, University of Essex \\ Edward Tsang \\ School of Computing and Electronic Systems \\ and Centre for Computational Finance and Economic Agents (CCFEA), University of Essex \\ Richard Olsen \\ Centre for Computational Finance and Economic Agents (CCFEA), University of Essex \\ and Olsen Ltd., Zurich \\ Alexandre Dupuis \\ Centre for Computational Finance and Economic Agents (CCFEA), University of Essex \\ and Olsen Ltd., Zurich \\ Please cite the corresponding journal article: \\ http://dx.doi.org/10.5018/economics-ejournal.ja.2012-36
}

\begin{abstract}
Financial markets witness high levels of activity at certain times, but remain calm at others. This makes the flow of physical time discontinuous. Therefore using physical time scales for studying financial time series, runs the risk of missing important activities. An alternative approach is the use of an event-based time that captures periodic activities in the market. In this paper, we use a special type of event, called a directional-change event, and show its usefulness in capturing periodic market activities. Our study confirms that the length of the price curve coastline as defined by directional-change events, turns out to be a long one.
\end{abstract}

Paper submitted to the special issue

New Approaches in Quantitative Modeling of Financial Markets

JEL G10

Keywords Directional-change event; intrinsic time; high-frequency finance; foreign exchange market; time-series analysis

Correspondence Monira Essa Aloud, School of Computer Science and Electronic Engineering, University of Essex, Colchester, Essex, United Kingdom; e-mail: memalo@essex.ac.uk 


\section{Introduction}

The Foreign Exchange (FX) market is open 24 hours a day, 7 days a week. Trading activities in FX market vary at different times of the day. For instance, on the announcement of political or economic news, there tends to be a sharp rise in market trading activity in response to the news, even during weekends when trading activity has a tendency to decline. Consequently, financial time series are unevenly spaced, which makes the flow of physical time discontinuous. This raises an essential need to redefine financial time series beyond the notion of physical time changes.

Mandelbrot and Taylor (1967) coined the term 'intrinsic time' in replace the notion of physical time when studying financial time series. Intrinsic time is defined by events, and events are characterised by a fixed threshold of different sizes. Events define time in price time series, eliminating any irrelevant details of price evolution. Guillaume et al. (1997) describe the price evolution by directional-change events frequency over a sampling period which provides an alternative measure of the risk. Glattfelder et al. (2011) introduce a directional-change events approach based on intrinsic time, where patterns emerge at different magnitudes for a sequence of events. Using statistical analysis based on the directional-change events approach, Glattfelder et al. (2011) discover 12 new empirical scaling laws related to foreign exchange data series across 13 currency exchange rates. These 12 scaling laws enhance our understanding of the behaviour of prices in financial markets, giving us new insights. Bisig et al. (2009) define the so-called Scale of Market Quakes (SMQ), designed based on directional-change events approach. The purpose of SMQ is to quantify FX market activity on a continuous basis at major economic and political events announcements.

In this paper we show the significance of the directional-change event approach for studying and analyzing financial time series. This is done using a 4 year data sample of high frequency tick-by-tick market data for EUR/USD and EUR/CHF, spanning January 1, 2006 to December 31, 2009. Our study shows that the length of the price-curve coastline defined by a directional-change events approach, is longer than the length of the price-curve coastline defined by fixed time intervals (physical time).

The remainder of the paper is organised as follows. Section 1 describes the concept of intrinsic time combined by showing empirically the limitations of a physical time scale for studying price time series. Section 2 gives a definition of the directional-change events approach. Data and empirical results, with regard directional-change events approach, are presented in Section 3. Section 4 offers a measurement of the length of EUR/USD price curve coastline.

\section{Intrinsic Time}

The majority of traditional approaches to observe price movements in financial time series are based on physical time changes. These are the changes that occur in a 


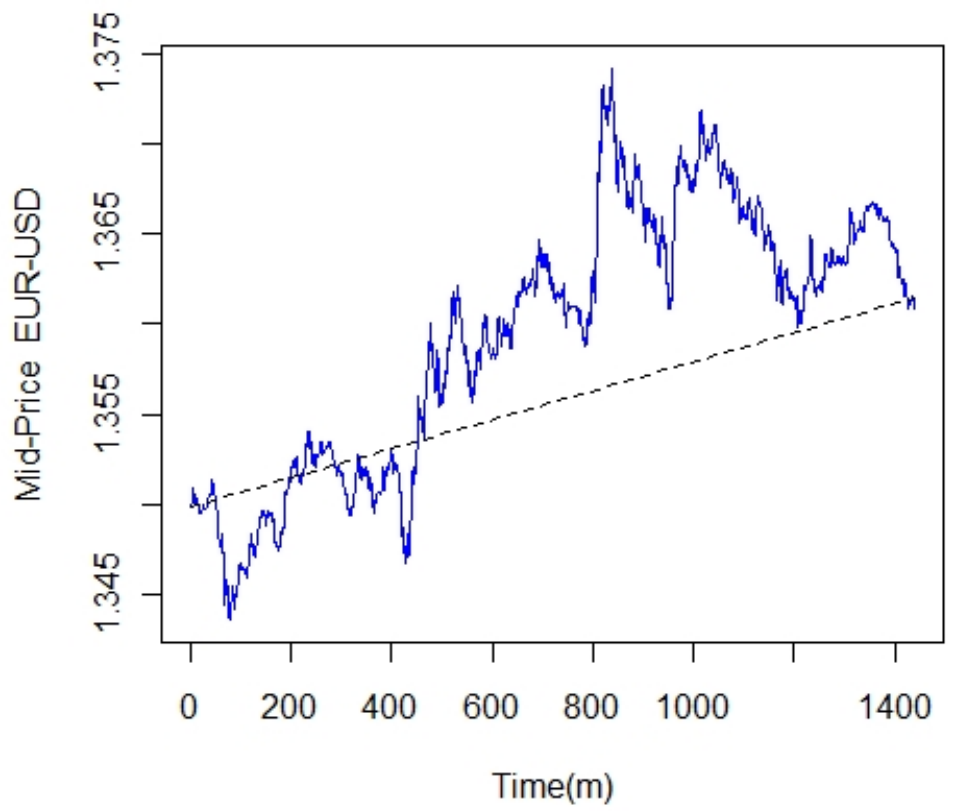

Figure 1: Price activities for EUR/USD on January 7, 2009. The straight line shows the gradient of the price changes for that day. The graph shows a major part of the activity is overlooked. So, chopping off the time series of prices will eliminate many significant price activities.

time series, ranging from seconds through hourly to daily changes, in which the flow of time is discontinuous. In addition, as a result of the availability of ultra high frequency data, in which data, by its own nature, is inhomogeneous in time, it has become increasingly difficult and challenging to observe price movements through the use of physical time (Dacorogna et al. 2001). Figures 1 and 2 show price activities for EUR/USD on January 7, 2009. From both graphs, it is clear that significant patterns of the trading activity are ignored when considering the time series of prices based on daily and hourly changes (physical time). By way of illustration, in Figure 1 and Figure 2, the price movement from midnight, hour 0, to hour 13 and 57 minutes represent an investment opportunity to sell, with the price rising by $1.8 \%$. This was overlooked in both the end of that day's return and the hourly return.

Physical time fails to clearly capture the full activity of price movements in a significant manner, since the variety of trading activity obviously depends on the time of day. Using physical time to detect periodic patterns in time series, maps a variety of patterns with different magnitudes, which makes the flow of physical time discontinuous. Extensive computational efforts are required to analyse and study price time series, which results in obvious costs associated with the study process. 


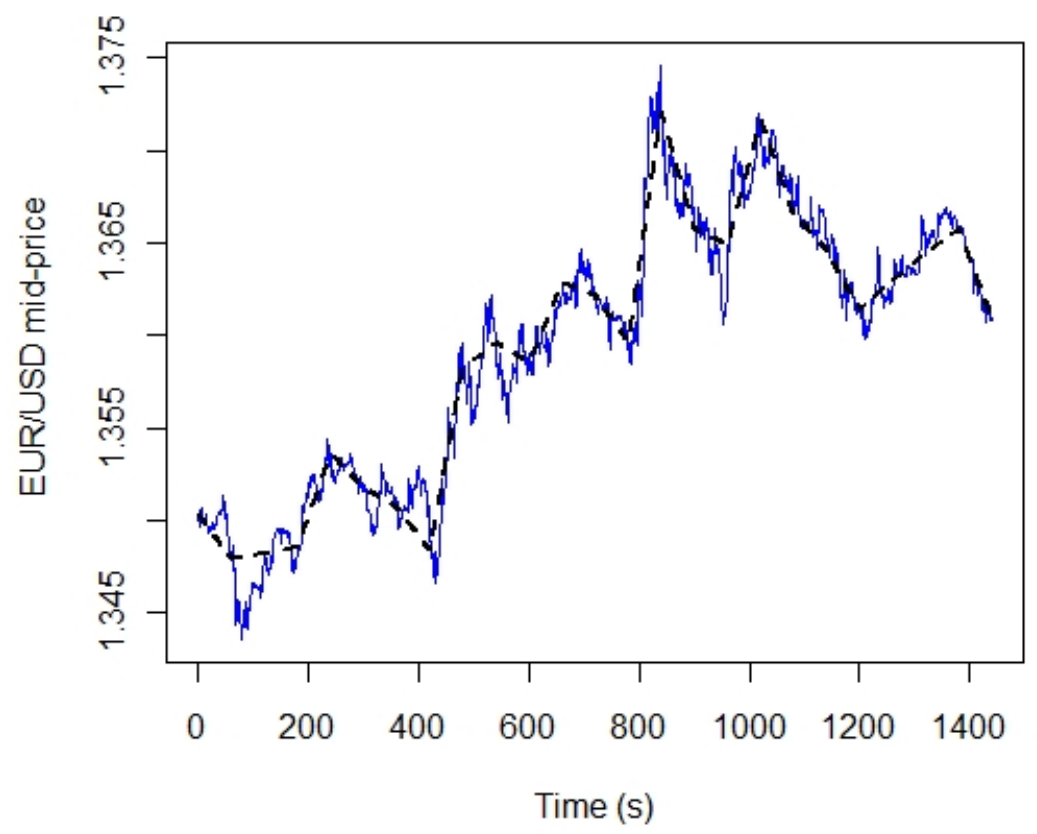

Figure 2: Price activities for EUR/USD on January 7, 2009. The dashed lines show hourly return for that day. Even with hourly return, some activity is still overlooked.

To deal with this vital issue, alternative solutions have been proposed. Among them is the study of financial time series using intrinsic time, which provides a reliable solution.

Intrinsic time adopts an event-based system in contrast to physical time which adopts a point-based system. In intrinsic time, time is defined by events. An event is characterized by a fixed threshold $\lambda$ and is defined as the absolute price change between two local extremal values exceeding a given threshold $\lambda$ (Glattfelder et al. 2011). Figure 3 shows EUR/USD price activities on the 7, January 2009 sample onto a reduced set of four sequential events defined by a threshold $\lambda=1 \%$. Physical time is homogenous which means time scales equally spaced on any chosen time scale. In contrast, intrinsic time is inhomogeneous in time seeing that time triggers only at periodic events, independent of the notion of physical time.

\section{Directional-change events approach}

\subsection{Directional-change event}

According to Tsang (2010) a directional-change event can take one of the two forms - a Downturn Event or an Upturn Event. A Downward Run is a period 


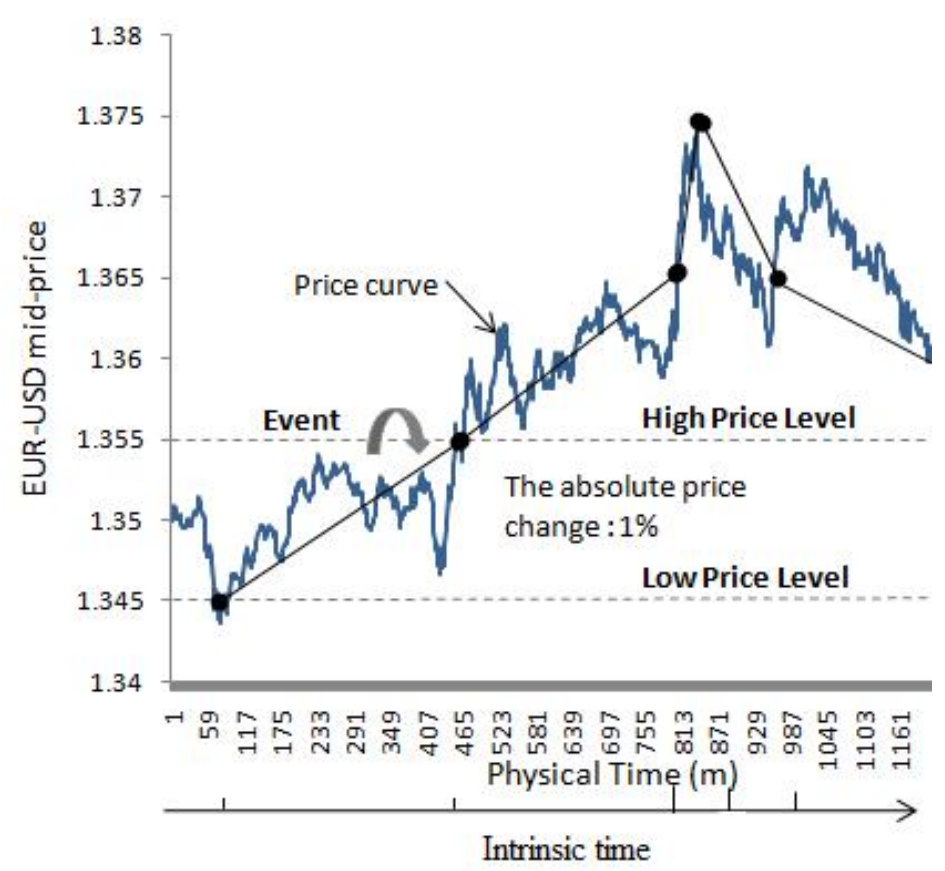

Figure 3: The graph shows a 24-hour EUR/USD mid-price sample and sequential events (solid lines) defined by a threshold $\lambda=1 \%$. Intrinsic time triggers only at periodic events whereas physical time ticks equally across different patterns of different magnitudes in the price curve.

between a Downturn Event and the next Upturn Event, while an Upward Run is a period between an Upturn Event and the next Downturn Event. A Downturn Event terminates an Upward Run, and starts a Downward Run, whereas an Upturn Event terminates a Downward Run and starts an Upward Run.

During a Downward Run, a Last Low price $p^{l}$ is continuously updated to the minimum of (a) the current market price $p(t)$ and (b) the Last Low price $p^{l}$. Similarity, during an Upward Run, a Last High price $p^{h}$ is continuously updated to the maximum of (a) the current market price $p(t)$ and (b) the Last High price $p^{h}$ (Tsang 2010). At the beginning of the sequence, the Last High price $p^{h}$ and Last Low price $p^{l}$ are set to the initial market price $p\left(t_{0}\right)$ at the beginning of the sequence (Tsang 2010).

In an Upward Run, a Downturn Event is an event when the absolute price change between the current market price $p(t)$ and the Last High price $p^{h}$ is lower than a fixed threshold $\triangle x_{d c}$

$$
p(t) \leq p^{h} *\left(1-\triangle x_{d c}\right)
$$

The starting point of a Downturn Event is a Downturn Point which is the point at which the price last peaked $\left(p^{h}\right)$. The end of a Downturn Event is a Downturn Directional-change Point which is the point at which the price has dropped from the last downturn point by the threshold $\triangle x_{d c}$ (Tsang 2010). 
In a Downward Run, an Upturn Event is an event when the absolute price change between the current market price $p(t)$ and the Last Low price $p^{l}$ is higher than a fixed threshold $\triangle x_{d c}$

$$
p(t) \geq p^{l} *\left(1+\triangle x_{d c}\right)
$$

The starting point of an Upturn Event is an Upturn Point which is the point at which the price last troughed $\left(p^{l}\right)$. The end of an Upturn Event is an Upturn Directional-change Point which is the point at which the price has risen from the last upturn point by the threshold $\triangle x_{d c}$.

\subsection{Overshoot event}

A directional-change (DC) event - a Downturn Event or an Upturn Event - is usually followed by a price overshoot event rather than an opposite directional-change event direction (Bisig et al. 2009). The overshoot (OS) event represents the time interval of price movement beyond the directional-change event. An overshoot event can take one of two forms - an Upward Overshoot or a Downward Overshoot event (Tsang 2010). An Upward Overshoot event is a period between the previous Upturn Event and the starting point of the next Downturn Event. Similarly, a Downward Overshoot event is a period between the previous Downturn Event and the starting point of the next Upturn Event. Figure 4 illustrates how the price curve is composed of directional-change and overshoot events. Algorithm 1 illustrates how to define directional change and overshoot events during a time period $T$.

In an Upward Run and a Downward Run, a total price movement $\triangle t_{T M}$ between two local extremal price values (minimum and maximum price) is decomposed into a directional change (DC) event and an overshoot (OS) event. A total price movement $\triangle t_{T M}$ is defined by

$$
\left|\triangle t_{T M}\right|=\left|\triangle t_{d c}\right|+\left|\triangle t_{o s}\right|
$$

In a Downward Run, a Downturn Event is usually followed by a Downward Overshoot Event, which is ended by the next Upturn Event. In an Upward Run, an Upturn Event is usually followed by an Upward Overshoot Event, which is ended by the next Downturn Event (Tsang 2010). So directional-change events approach define price time series as a sequence of

$\cdots \rightarrow$ Downurn Event $\rightarrow$

Downward Overshoot Event $\rightarrow$

Upturn Event $\rightarrow$

Upward Overshoot Event $\rightarrow$

Downurn Event $\rightarrow \cdots$ 


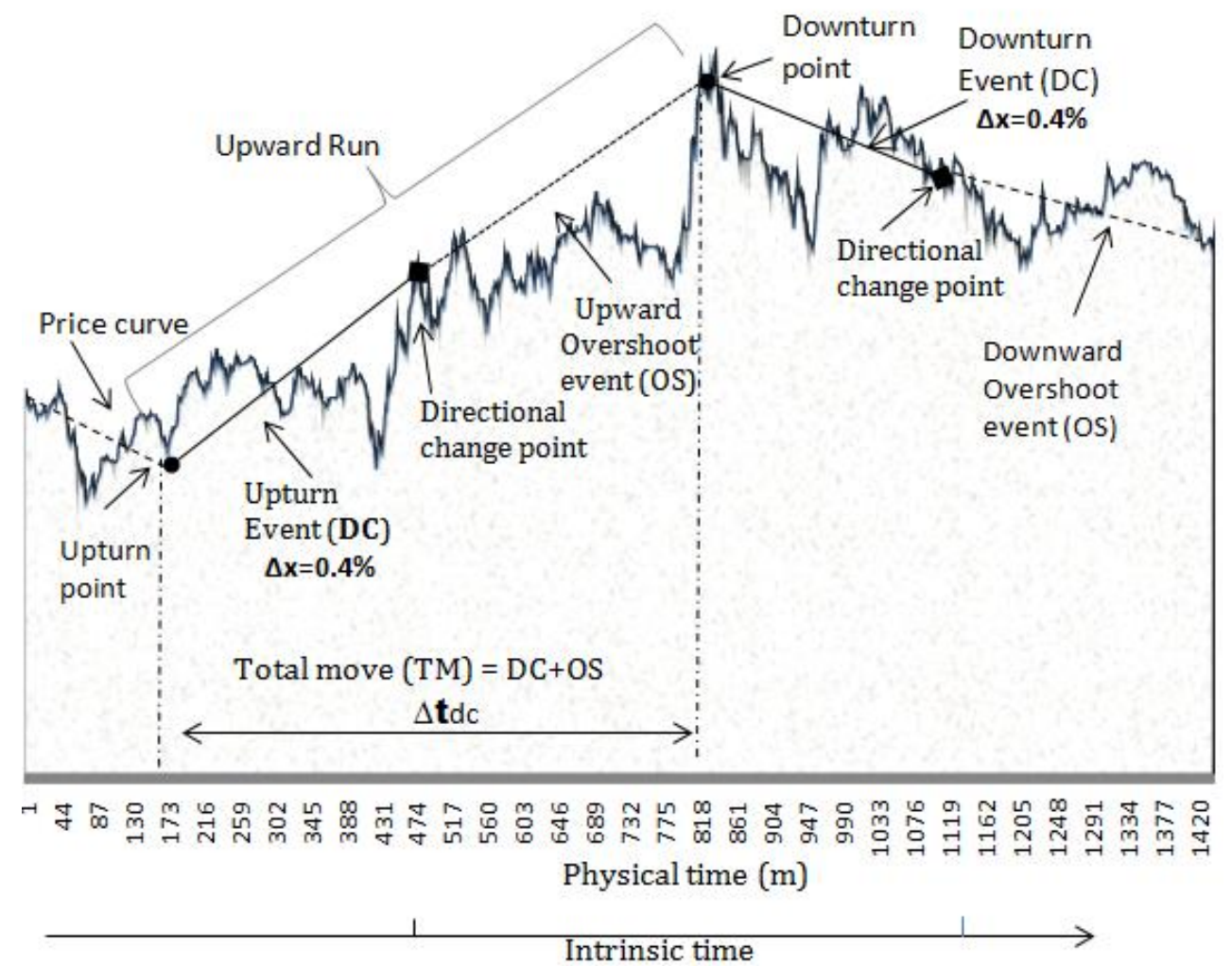

Figure 4: Price movements within 24 hours are shaped by a directional-change points (diamond) with a threshold $\triangle x_{d c}=0.4 \%$. A total price movement between two local values (minimum and maximum price) is decomposed into directional change (solid lines) and an overshoot (dashed lines) events.

\section{Spectral Analysis of Tick Data}

\subsection{The Dataset}

We use a high-frequency database composed of two currency pairs (EUR/USD and EUR/CHF) spanning a four year period, from January 1, 2006 to December 31, 2009. The data set includes a bid, an ask rate of a currency pair at a timestamp. Throughout the paper, the following definition of mid-price is used

$$
p(t)=\frac{\left(B_{i}+A s k_{t}\right)}{2}
$$

where $p(t)$ is the mid-price of a currency pair at time $t, B_{i d}$ is the bid rate at time $t$ and $A s k_{t}$ is the ask rate at time $t$. We aim to use this high-frequency dataset to show the significance of directional-change events in capturing periodic market activities. 


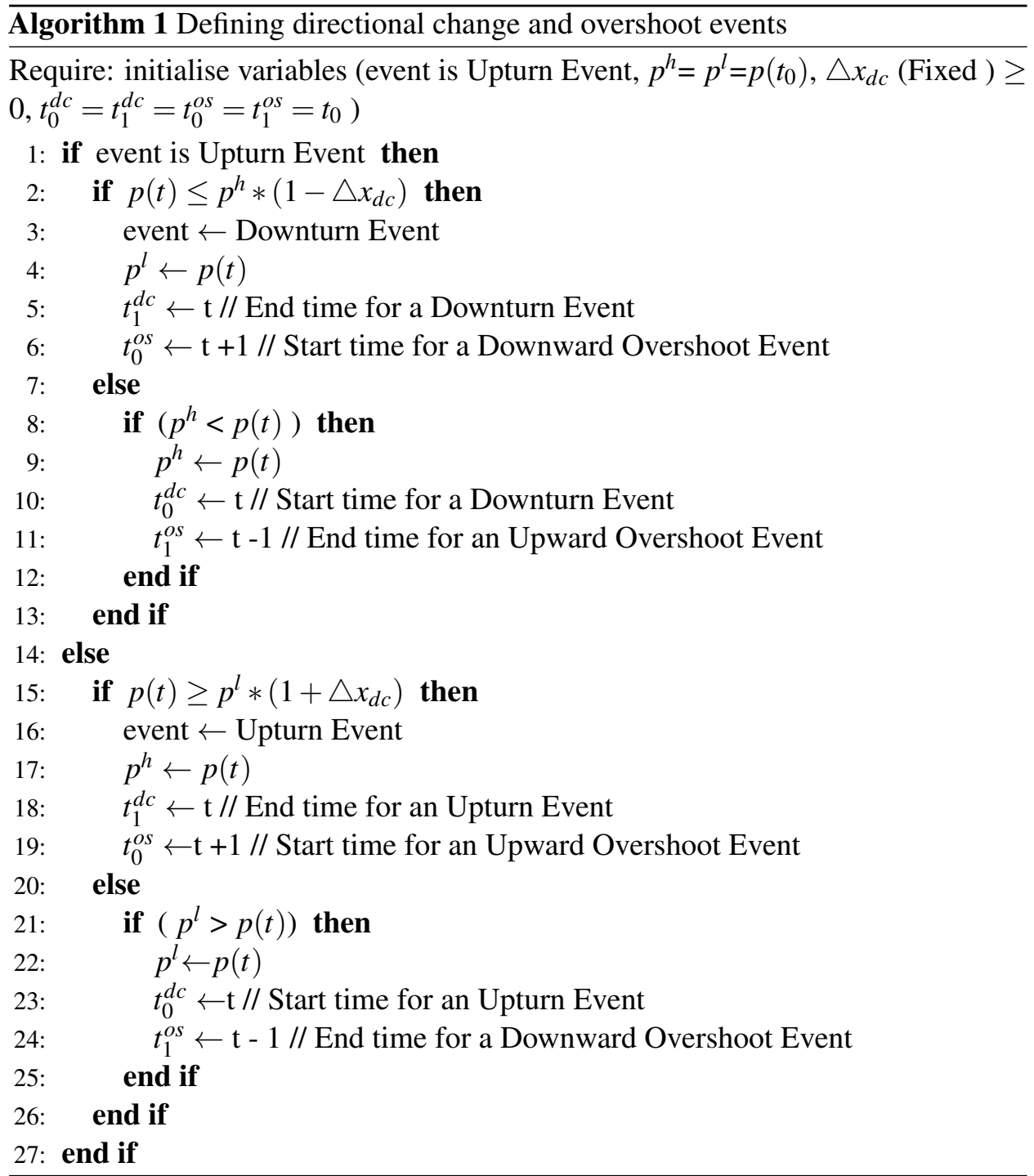

\subsection{Event Time Scales}

Directional-change events are significant events seeing that they capture periodic major change in a price time series in which the magnitude of an event is defined by the observer. Under physical time, we divide time into periods of equal length which has the drawback of missing significant events in a price time series. However, different time periods in a price time series may contain different number of events of different magnitude, which demonstrate the fact of price evolution in isolation of physical time changes.

Figures 5 and 6 report the number of events of $0.03 \%$ and $0.10 \%$ magnitude in different time periods of the $5^{\text {th }}, 7^{\text {th }}$ and $9^{\text {th }}$ January 2009 in EUR/USD and EUR/CHF mid-price time series. For EUR/USD, we can observe from the results in 


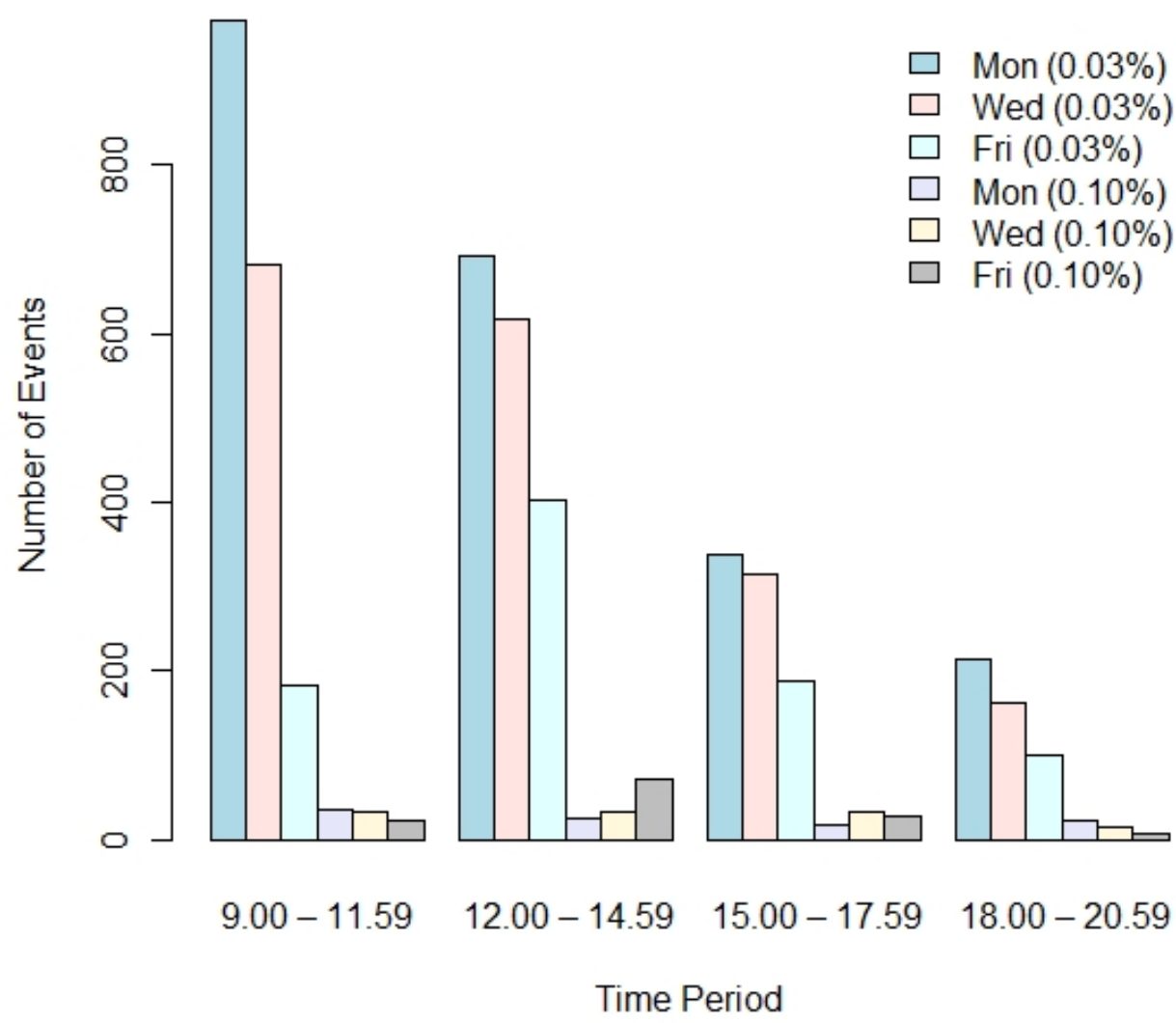

Figure 5: Number of events of $0.03 \%$ and $0.10 \%$ magnitude in different periods of the $5^{\text {th }}$ (Monday), $7^{\text {th }}$ (Tuesday) and $9^{\text {th }}$ (Friday) January 2009 in EUR/USD mid-price time series.

Figure 5, that the period between 9:00 - 11:59 on the 5th January contained 971 events with a threshold of $0.03 \%$, while for the same period on the 7th January there were 683 events. On the 7th January there were 34 events for three periods of time with a $0.10 \%$ threshold, while this was not the case on the 5th January. Obviously, the reported results in Figures5 and 6 pin-pointed tow important observations: (a) for different currency pairs the same periods of time with the same threshold size on different days may contain a different number of events, and (b) with the same threshold size, some periods on the same day have more events than others. These two observations indicate that events of different magnitude are independent of physical-time changes. 


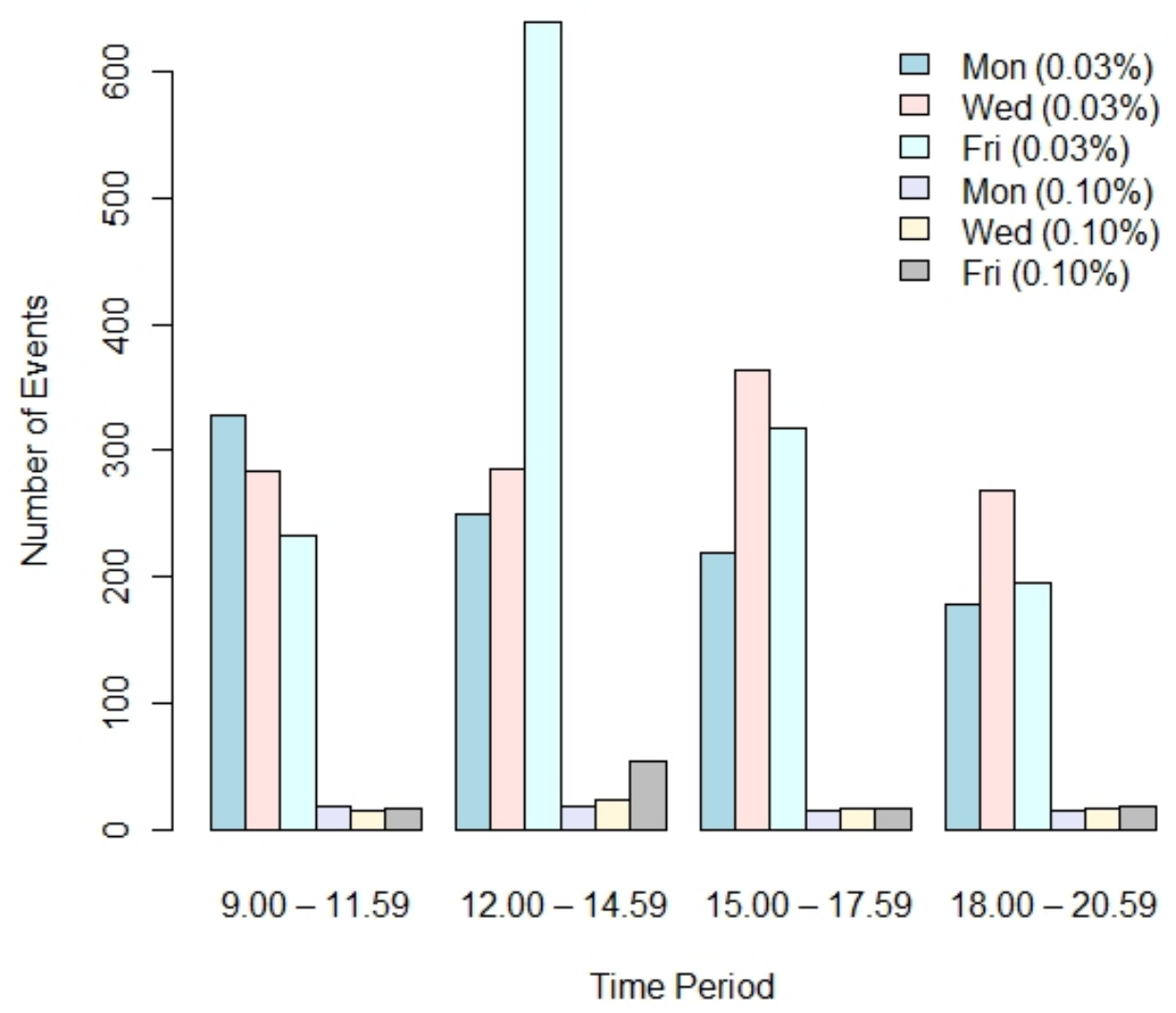

Figure 6: Number of events of $0.03 \%$ and $0.10 \%$ magnitude in different periods of the $5^{\text {th }}$ (Monday), $7^{\text {th }}$ (Tuesday) and $9^{\text {th }}$ (Friday) January 2009 in EUR/CHF mid-price time series.

\section{The price-curve coastline}

In this section a measurement of the length of the price-curve coastline is conducted, based on the price distance between fixed points. The measurement is defined by two different concepts: intrinsic time (directional-change events) and physical time changes at fixed time intervals, with the aim of assessing their performance in summarizing price movements. Assuming prefect foresight, the length of the price-curve coastline over a defined time period $T$, represents the profit potential. Glattfelder et al. (2011) discovered scaling laws that give an estimation of the length of the price curve coastline. They found that the length of the coastline defined by intrinsic time is long.

The measurement of the length of the price-curve coastline over a time period $T$, as defined by intrinsic time, is the average upwards and downwards price moves, 
considering the number of events. Both the upwards and downwards price movements are defined by a fixed threshold size $\triangle x_{d c}$. The downward price movement is the total price move from a Downturn Point to the next Upturn Point. In another word, the downturn price movement is the absolute difference between $p_{i}$ and $p_{i+1}$, where $p_{i}$ is the price of the $\mathrm{i}$-th downturn turning point and $p_{i+1}$ is the price of the next upturn point. Similarly, the upward price movement is the total price move from an Upturn Point to the next Downturn Point. Under intrinsic time, the length of the price-curve coastline $C\left(\triangle x_{d c}\right)$ is measured by

$$
c\left(\triangle x_{d c}\right)=\frac{1}{N\left(\triangle x_{d c}\right)} \sum_{i=1}^{N\left(\triangle x_{d c}\right)}\left|p_{i}-p_{i+1}\right|
$$

where $\triangle x_{d c}$ is a fixed threshold $(\%), N\left(\triangle x_{d c}\right)$ is the total number of events on which the length of the price-curve coastline is measured. Hence, the number of events is determined by the threshold size, $\triangle x_{d c}$, that we use. $p_{i}$ is the price of the i-th turning point, whether upturn or downturn point.

In contrast, the measurement of the length of the price-curve coastline defined by physical time changes at fixed time intervals is the average price movement between fixed points over a time period $T$, in which the time interval between these fixed points is equivalent. Under physical time, the length of the price-curve coastline $C\left(\triangle x_{d c}\right)$ is measured by

$$
c(t)=\frac{1}{n} \sum_{i=1}^{n}\left|p_{i}-p_{i+1}\right|
$$

where $p_{i}$ is the price of point $n$ and $n$ is the total number of fixed points which is equal to the number of events used in equation 5. For a fair comparison, we use the same number of points in physical time and intrinsic time.

Figure 7 shows EUR/USD mid-price activities over November $5^{\text {th }}, 2009$. The graph contrasts the price changes defined by physical time and by intrinsic time using the same number of points. The lines in the graph summarize the price movements using intrinsic time and physical time (at fixed time intervals). The price changes under intrinsic time are represented in the graph by straight lines lining up the Upturn/Downturn Points in terms of directional change events. From figure 7, it is obvious that significant events of trading activity are ignored when we consider changes over time based on fixed time intervals (physical time changes). By way of illustration, the price peak at 1.4917 was overlooked on the price curve based on physical time changes. Therefore, the length of the coastline defined by physical time $(0.01 \%)$ is shorter than the coastline defined by intrinsic time $(0.10 \%)$ which highlights the significance of intrinsic time.

It is important to be acquainted with how well intrinsic time and physical time lines fit prices in a time series. This allows the evaluation of their performance in terms of which is finest at describing the price changes in a time series. We measure the total error, $E_{T}$, of the length of the price curve coastlines under both physical 


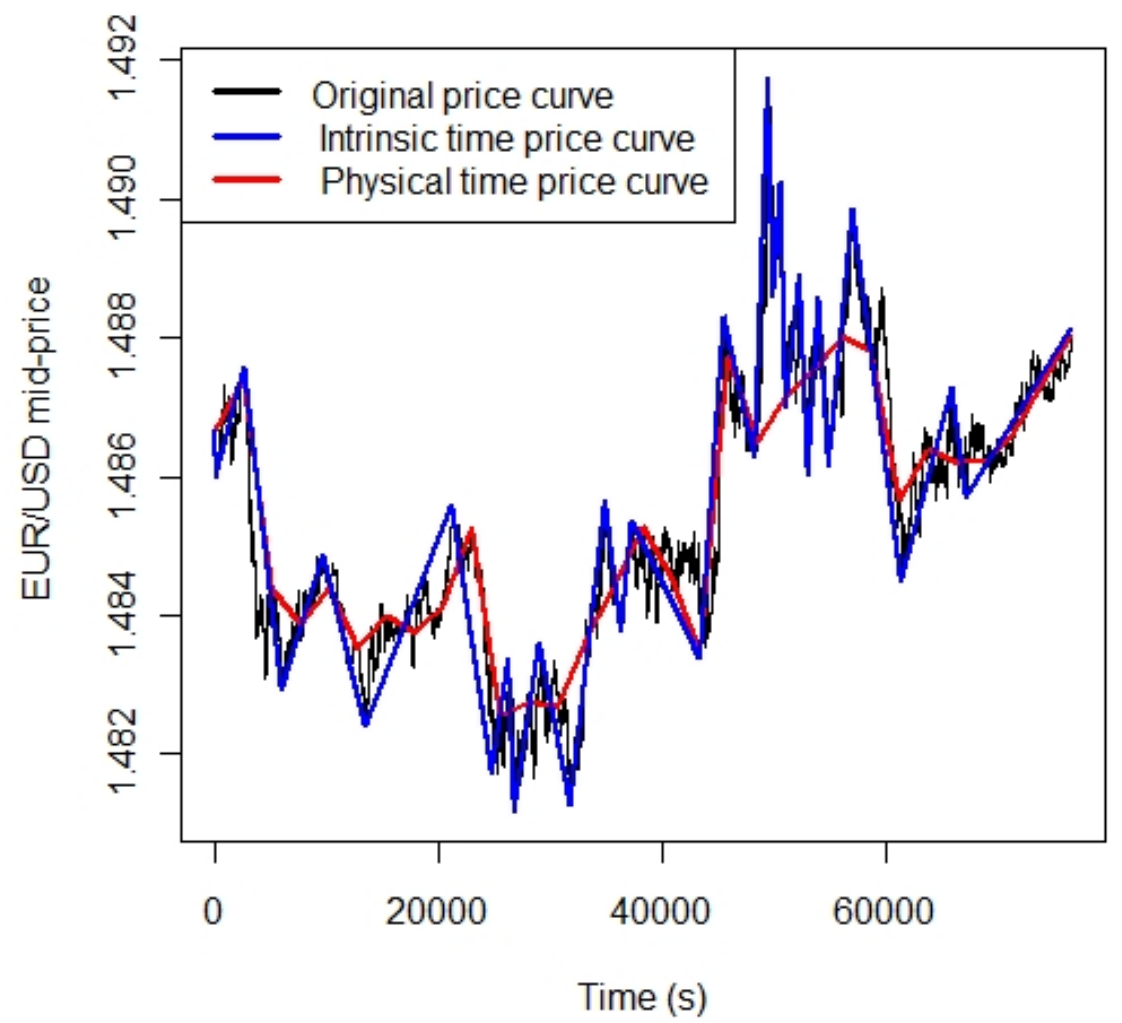

Figure 7: EUR/USD mid-prices time series defined by physical time and intrinsic time. The sampling period is over November $5^{\text {th }}, 2009$. For intrinsic time, the number of events is 30 determined by a threshold size of $0.1 \%$.

time and intrinsic time conditions (Figure 7). The total error, $E_{T}$, is a measure of the overall error of intrinsic time and physical time in summarizing price movement over a time period $T$, and is defined by

$$
E_{T}=\sum_{t=0}^{n}\left(\triangle x_{t}\right)^{2}
$$

where $n$ is the total number of time scales over a time period $T,\left(\triangle x_{t}\right)^{2}$ is the square difference between two prices in the price curve and in the physical timelintrinsic time line calculated for the same time scale, $t$. We found that the measurement of the total error in terms of intrinsic time (0.03) is shorter than that for physical time (0.04) (Figure 7). This result indicates that summarizing price movements using intrinsic time gives a better description of price changes than does using physical time with fixed intervals. 


\begin{tabular}{|c|c|c|c|c|}
\hline Threshold & $\overline{N(\text { points })}$ & $\overline{P T I(\sec )}$ & $c\left(\triangle x_{d c}\right)$ & $c(t)$ \\
\hline $0.01 \%$ & 545,793 & 84 & $0.04 \%$ & $0.01 \%$ \\
\hline $0.10 \%$ & 11,870 & 3,973 & $0.47 \%$ & $0.08 \%$ \\
\hline $1 \%$ & 778 & 83,239 & $3.15 \%$ & $0.37 \%$ \\
\hline $3 \%$ & 189 & 876,365 & $7 \%$ & $1.16 \%$ \\
\hline
\end{tabular}

Table 1: The measurement of the length of EUR/USD price-curve coastline over four years from 2006 to 2009 , as defined by intrinsic time (directional-change events) $c\left(\triangle x_{d c}\right)$ and physical time changes (fixed intervals) $c(t)$. The average number of fixed points is denoted by $\overline{N(\text { points })}$ and the fixed time interval in physical time series is denoted by $\overline{P T I(\mathrm{sec})}$.

\begin{tabular}{|c|c|c|c|c|}
\hline Threshold & $\overline{N(\text { points })}$ & $\overline{P T I(s e c)}$ & $c\left(\triangle x_{d c}\right)$ & $c(t)$ \\
\hline $0.01 \%$ & 698,914 & 88 & $0.04 \%$ & $0.01 \%$ \\
\hline $0.10 \%$ & 7,196 & 10,657 & $0.56 \%$ & $0.07 \%$ \\
\hline $1 \%$ & 410 & 129,100 & $3.11 \%$ & $0.29 \%$ \\
\hline $3 \%$ & 30 & $2,318,338$ & $5.87 \%$ & $1.12 \%$ \\
\hline
\end{tabular}

Table 2: The measurement of the length of EUR/CHF price-curve coastline over four years from 2006 to 2009 , as defined by intrinsic time (directional-change events) $c\left(\triangle x_{d c}\right)$ and physical time changes (fixed intervals) $c(t)$. The average number of fixed points is denoted by $\overline{N(\text { points })}$ and the fixed time interval in physical time series is denoted by $\overline{P T I(s e c)}$.

The reported results in Table 1 and 2 compare the average lengths of the annualized EUR/USD and EUR/CHF coastline measured by (a) physical time scales (evenly spaced in time - fixed time interval), and (b) intrinsic time (unevenly spaced in time). The sampling period covers the four years from 2006 to 2009. We annualize the results by dividing them by 4 , the number of years in our data sample.The measurement of the length of EUR/USD and EUR/CHF price-curve coastline over each of the four years is in appendix A.

The results in Table 1 and 2 draw attention to the fact that the length of the coastline depends completely on the frequency of changes in the price. Also, these reported results highlight the importance of considering events in studying the price-curve, rather than physical time changes due to the long coastline of price changes based on intrinsic time. In all the thresholds that we have tested, the length of the price-curve coastline defined by physical time is shorter than the coastline defined by intrinsic time. Intrinsic time enables the analyst to capture the short term market dynamics involved by presenting significant information and a clear picture of price behaviour, based on the observer's expectations of the market. In addition, it reduces the complexity of real-world price time series given the small number of price points for evaluation. The computational costs (which include the cost of evaluating data) must not be ignored. Thus, it should be part of the criteria when it comes to deciding on an approach for studying price time series. 


\section{Conclusion}

Directional-change events approach can gradually improve our understanding of the dynamic behaviour of financial markets in a simplified way, beyond what physical time at fixed time intervals can achieve. This approach allows the understanding of the price time series as an event-based process independent of the notion of physical time which allows the detection of periodic patterns.

This paper illustrates the definition of directional-change events approach in conjunction with the motivation behind introducing this approach, its advantages and significance, and the working mechanisms of this approach. The spectral analysis shows that the size of the threshold determines the number of events in a price time series. An increase in the size of the threshold, $\Delta x_{d c}$, means a decrease in the number of existence events in the price time series. Additionally, the reported results in the paper indicate that with the same threshold size the same periods of time on different days may contain a different number of events. Also, with the same threshold value, some periods on the same day may have more events than others. The measurement of the length of the price-curve coastline shows a long coastline of price changes defined by intrinsic time confirming similar results in Glattfelder et al. (2011) study.

The directional-change events approach opens doors to many research directions on financial markets. One obvious direction is to extend the catalogue of stylised facts by studying the dynamic behaviour of markets based on directional-change events approach. Another important research direction is to model a trading strategy based on directional-change events. This would allow traders to capture the short term market dynamics involved by the detection of major periodic patterns in time series.

We believe that directional-change events approach has the potential to act as a robust foundation for studying financial time series, and could lead to new findings about financial behaviour in various situations.

\section{Acknowledgment}

The authors would like to thank Olsen Ltd for their help and for providing the FX market data. This work was supported in part by the Olsen Ltd.

\section{References}

Bisig, T., Dupuis, A., Impagliazzo, V., and R. Olsen (2009) .The scale of market quakes. Working paper, URL: arXiv:0909.1690v1.

Dacorogna, M. M., Gen, cay, R., M“uller, U. A., Olsen, R. B, and O. V. Pictet (2001). An introduction to high-frequency finance. Academic Press: San Diego. 
Glattfelder, J. B., Dupuis, A., and R. B. Olsen (2011). Patterns in high-frequency FX data: discovery of 12 empirical scaling laws. Quantitative Finance 11(4): 599-614. Guillaume, D. M., Dacorogna, M. M., Davé,R. R., Müller,U. A., Olsen, R. B., and O. V. Pictet (1997). From the bird's eye to the microscope: A survey of new stylized facts of the intra-daily foreign exchange markets. Finance and Stochastics 1(2): 95-129.

Mandelbrot, B., and H. M. Taylor (1967). On the distribution of stock prices differences. Operations Research 15(6): 1057-1062.

Tsang, E.P.K. (2010). Directional changes, definitions. Working Paper WP050-10, Centre for Computational Finance and Economic Agents (CCFEA), University of Essex, URL: http://www.essex.ac.uk/ccfea/research/WorkingPapers/2010/CCFEA\%20WP05010.pdf

\section{A appendix}

\begin{tabular}{ccccc}
\hline Threshold & N(points) & PTI $(\mathrm{sec})$ & $c\left(\triangle x_{d c}\right)$ & $c(t)$ \\
\hline \hline $0.01 \%$ & 257,188 & 122 & $0.04 \%$ & $0.01 \%$ \\
$0.10 \%$ & 5,775 & 5,419 & $0.37 \%$ & $0.07 \%$ \\
$1 \%$ & 288 & 108,664 & $2.65 \%$ & $0.40 \%$ \\
$3 \%$ & 41 & 763,298 & $5.11 \%$ & $1 \%$ \\
\hline
\end{tabular}

Table 3: The measurement of the length of EUR/USD price-curve coastline over 2006, as defined by intrinsic time (directional-change events) $c\left(\triangle x_{d c}\right)$ and physical time changes (fixed intervals) $c(t)$. The number of fixed points denoted by $\mathrm{N}$ (points) and the fixed time interval in physical time series denoted by PTI (sec).

\begin{tabular}{ccccc}
\hline Threshold & N(points) & PTI $(\mathrm{sec})$ & $c\left(\triangle x_{d c}\right)$ & $c(t)$ \\
\hline \hline $0.01 \%$ & 227,400 & 188 & $0.04 \%$ & $0.01 \%$ \\
$0.10 \%$ & 4,439 & 7,085 & $0.39 \%$ & $0.07 \%$ \\
$1 \%$ & 181 & 173,763 & $2.59 \%$ & $0.45 \%$ \\
$3 \%$ & 13 & $2,419,318$ & $8.15 \%$ & $2.02 \%$ \\
\hline
\end{tabular}

Table 4: The measurement of the length of EUR/USD price-curve coastline over 2007, as defined by intrinsic time (directional-change events) $c\left(\triangle x_{d c}\right)$ and physical time changes (fixed intervals) $c(t)$. The number of fixed points denoted by $\mathrm{N}$ (points) and the fixed time interval in physical time series denoted by PTI (sec). 


\begin{tabular}{ccccc}
\hline Threshold & N(points) & PTI $(\mathrm{sec})$ & $c\left(\triangle x_{d c}\right)$ & $c(t)$ \\
\hline \hline $0.01 \%$ & 882,014 & 36 & $0.04 \%$ & $0.01 \%$ \\
$0.10 \%$ & 19,295 & 1,635 & $0.67 \%$ & $0.08 \%$ \\
$1 \%$ & 1,636 & 19,284 & $4.79 \%$ & $0.29 \%$ \\
$3 \%$ & 585 & 53,930 & $8.96 \%$ & $0.51 \%$ \\
\hline
\end{tabular}

Table 5: The measurement of the length of EUR/USD price-curve coastline over 2008, as defined by intrinsic time (directional-change events) $c\left(\triangle x_{d c}\right)$ and physical time changes (fixed intervals) $c(t)$. The number of fixed points denoted by $\mathrm{N}$ (points) and the fixed time interval in physical time series denoted by PTI (sec).

\begin{tabular}{ccccc}
\hline Threshold & N(points) & PTI $(\mathrm{sec})$ & $c\left(\triangle x_{d c}\right)$ & $c(t)$ \\
\hline \hline $0.01 \%$ & 816,568 & 39 & $0.04 \%$ & $0.01 \%$ \\
$0.10 \%$ & 17,969 & 1,751 & $0.43 \%$ & $0.08 \%$ \\
$1 \%$ & 1,007 & 31,244 & $2.58 \%$ & $0.34 \%$ \\
$3 \%$ & 117 & 268,914 & $5.77 \%$ & $1.10 \%$ \\
\hline
\end{tabular}

Table 6: The measurement of the length of EUR/USD price-curve coastline over 2009, as defined by intrinsic time (directional-change events) $c\left(\triangle x_{d c}\right)$ and physical time changes (fixed intervals) $c(t)$. The number of fixed points denoted by $\mathrm{N}$ (points) and the fixed time interval in physical time series denoted by PTI (sec).

\begin{tabular}{ccccc}
\hline Threshold & N(points) & PTI $(\mathrm{sec})$ & $c\left(\triangle x_{d c}\right)$ & $c(t)$ \\
\hline \hline $0.01 \%$ & 191,533 & 163 & $0.03 \%$ & $0.01 \%$ \\
$0.10 \%$ & 1,200 & 26,047 & $0.61 \%$ & $0.08 \%$ \\
$1 \%$ & 105 & 29,7681 & $3.93 \%$ & $0.35 \%$ \\
$3 \%$ & 7 & $4,465,214$ & $5.30 \%$ & $1.16 \%$ \\
\hline
\end{tabular}

Table 7: The measurement of the length of EUR/CHF price-curve coastline over 2006, as defined by intrinsic time (directional-change events) $c\left(\triangle x_{d c}\right)$ and physical time changes (fixed intervals) $c(t)$. The number of fixed points denoted by $\mathrm{N}$ (points) and the fixed time interval in physical time series denoted by PTI (sec).

\begin{tabular}{ccccc}
\hline Threshold & N(points) & PTI $(\mathrm{sec})$ & $c\left(\triangle x_{d c}\right)$ & $c(t)$ \\
\hline \hline $0.01 \%$ & 233,820 & 135 & $0.04 \%$ & $0.01 \%$ \\
$0.10 \%$ & 2,744 & 11,462 & $0.73 \%$ & $0.07 \%$ \\
$1 \%$ & 354 & 88,845 & $3.10 \%$ & $0.24 \%$ \\
$3 \%$ & 10 & $3,145,106$ & $5.32 \%$ & $1.19 \%$ \\
\hline
\end{tabular}

Table 8: The measurement of the length of EUR/CHF price-curve coastline over 2007, as defined by intrinsic time (directional-change events) $c\left(\triangle x_{d c}\right)$ and physical time changes (fixed intervals) $c(t)$. The number of fixed points denoted by $\mathrm{N}$ (points) and the fixed time interval in physical time series denoted by PTI (sec). 


\begin{tabular}{ccccc}
\hline Threshold & N(points) & PTI (sec) & $c\left(\triangle x_{d c}\right)$ & $c(t)$ \\
\hline \hline $0.01 \%$ & $1,003,089$ & 31 & $0.04 \%$ & $0.01 \%$ \\
$0.10 \%$ & 13,595 & 2,321 & $0.49 \%$ & $0.07 \%$ \\
$1 \%$ & 839 & 37,609 & $3.05 \%$ & $0.31 \%$ \\
$3 \%$ & 78 & 404,538 & $7.18 \%$ & $1.17 \%$ \\
\hline
\end{tabular}

Table 9: The measurement of the length of EUR/CHF price-curve coastline over 2008, as defined by intrinsic time (directional-change events) $c\left(\triangle x_{d c}\right)$ and physical time changes (fixed intervals) $c(t)$. The number of fixed points denoted by $\mathrm{N}$ (points) and the fixed time interval in physical time series denoted by PTI (sec).

\begin{tabular}{ccccc}
\hline Threshold & N(points) & PTI (sec) & $c\left(\triangle x_{d c}\right)$ & $c(t)$ \\
\hline \hline $0.01 \%$ & $1,367,212$ & 23 & $0.03 \%$ & $0.01 \%$ \\
$0.10 \%$ & 11,244 & 2,798 & $0.40 \%$ & $0.05 \%$ \\
$1 \%$ & 341 & 92,265 & $2.36 \%$ & $0.27 \%$ \\
$3 \%$ & 25 & $1,258,495$ & $5.66 \%$ & $0.96 \%$ \\
\hline
\end{tabular}

Table 10: The measurement of the length of EUR/CHF price-curve coastline over 2009, as defined by intrinsic time (directional-change events) $c\left(\triangle x_{d c}\right)$ and physical time changes (fixed intervals) $c(t)$.The number of fixed points denoted by $\mathrm{N}$ (points) and the fixed time interval in physical time series denoted by PTI (sec). 


\section{Conomics}

The Open-Access, Open-Assessment E-Journal

Please note:

You are most sincerely encouraged to participate in the open assessment of this discussion paper. You can do so by either recommending the paper or by posting your comments.

Please go to:

http://www.economics-ejournal.org/economics/discussionpapers/2011-28

The Editor 